Hier steht eine Anzeige.

Springer

Enttäuschung an der Beeren-Front

\title{
Cranberrys bringen nichts beim Harnwegsinfekt
}

Ein weiteres Mal haben sich Forscher in einem Cochrane-Review den Wirkungen von Cranberrys gegen Harnwegsinfektionen (HWI) gewidmet. Ihr Urteil fällt negativ aus.

2008 hatte es noch nach einem geringen Nutzen von Cranberry-Saft zumindest bei Frauen mit rezidivierenden HWI ausgesehen. Seither sind die Ergebnisse von 14 weiteren Studien mit dem Beerensaft veröffentlicht worden und in den neuesten Cochrane-Review eingeflossen. Das Resümee von Studienleiterin Ruth Jepsen, University of Stirling, fällt nun noch pessimistischer aus: „Jetzt, da wir unseren Review mit weiteren Studien aktualisiert haben, legen die Ergebnisse nahe, dass
Cranberry-Saft sogar noch weniger vorbeugende Wirkung gegen Harnwegsinfekte hat als im Update davor."

In keiner der untersuchten Gruppen konnte ein überzeugender Nutzen im Vergleich zu Placebo, Antibiotika, Laktobazillen oder keiner Kontrolltherapie nachgewiesen werden. Zu den insgesamt 4473 Probanden in 24 Studien gehörten, neben den erwähnten Frauen mit wiederkehrenden HWI, weitere Risikogruppen: ältere Frauen und Männer, katheterisierte Patienten, Schwangere, Patienten mit Anomalien des Harntrakts und Kinder, die einen ersten oder rezidivierenden Infekt erlitten hatten.

Jepson RG et al, Cochrane Database Syst Rev 2012

Zwei Studien belegen gewichtiges Problem

\section{Dicke Kinder brechen sich häufiger die Knochen}

Kinder, die für ihr Alter zu viele Pfunde auf die Waage bringen, brechen sich häufiger das Bein als normalgewichtige Altersgenossen. Gleich zwei Teams von Wissenschaftlern aus den USA und Italien kommen in ihren Studien zu diesem Ergebnis.

Das Risiko einer Fraktur an Fuß, Knöchel, Unterschenkel oder im Kniebereich stieg in einer US-amerikanischen Untersuchung bei Kindern bereits ab einem BMI von $25 \mathrm{~kg} / \mathrm{m}^{2}$ stetig an. Leicht übergewichtige Kinder hatten ein um $17 \%$ erhöhtes Risiko gegenüber normalgewichtigen Altersgenossen, bei extrem adipösen (BMI $\geq$ $30 \mathrm{~kg} / \mathrm{m}^{2}$ ) war die Wahrscheinlichkeit eines Knochenbruchs an der unteren Extremität bis einschließlich zum Knie bereits um $45 \%$ erhöht. Die Studie von Jeff Kessler und Kollegen beruht auf Daten von fast

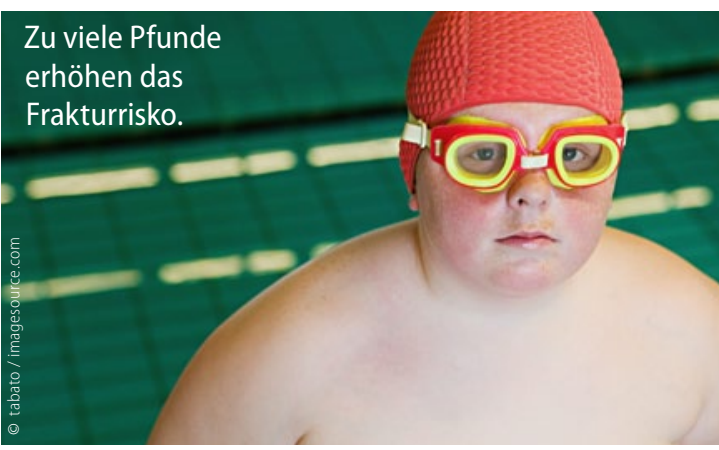

1 Mio. Kindern im Alter zwischen zwei und 19 Jahren, die an einer großen Kohortenstudie in Südkalifornien, der Kaiser Permanente Children's Health Study, teilgenommen hatten.

Kessler J et al, Clin Orthop Relat Res 2012

Valerio G et al, BMC Pediatr 2012 (online first) 